OPEN ACCESS

Edited by:

Jun Ren,

University of Washington,

United States

Reviewed by:

Christian Cadeddu Dessalvi,

University of Cagliari, Italy

Naifeng Liu,

Southeast University, China

${ }^{*}$ Correspondence:

Wenliang Zha

xyzW/800@163.com

Wei Yu

yuwei0805@163.com

${ }^{\dagger}$ These authors have contributed equally to this work

Specialty section:

This article was submitted to

Vascular Physiology,

a section of the journal

Frontiers in Physiology

Received: 21 December 2019

Accepted: 07 April 2020

Published: 19 May 2020

Citation:

Yang Z, Wang $M$, Zhang Y, Cai F, Jiang B, Zha W and Yu W (2020)

Metformin Ameliorates Diabetic

Cardiomyopathy by Activating

the PK2/PKR Pathway.

Front. Physiol. 11:425.

doi: 10.3389/fphys.2020.00425

\section{Metformin Ameliorates Diabetic Cardiomyopathy by Activating the PK2/PKR Pathway}

\author{
Zhen Yang ${ }^{1+}$, Min Wang ${ }^{1+}$, Yuchen Zhang ${ }^{1}$, Fei Cai' ${ }^{2}$, Botao Jiang ${ }^{3}$, Wenliang Zha ${ }^{4,5 *}$ and \\ Wei Yu${ }^{1 *}$
}

\begin{abstract}
${ }^{1}$ Department of Pharmacology, School of Pharmacy, Hubei University of Science and Technology, Xianning, China, ${ }^{2}$ Hubei Province Key Laboratory on Cardiovascular, Cerebrovascular, and Metabolic Disorders, Hubei University of Science and Technology, Xianning, China, ${ }^{3}$ Department of Urology, Xianning Central Hospital, Xianning, China, ${ }^{4}$ Department of Surgery, Clinic Medical College, Hubei University of Science and Technology, Xianning, China, ${ }^{5}$ National Demonstration Center for Experimental General Medicine Education, Hubei University of Science and Technology, Xianning, China
\end{abstract}

Diabetic cardiomyopathy (DCM) is a complication of diabetes that can cause damage to myocardial structure and function. Metformin (Met) is a widely used type 2 diabetes treatment drug that exerts cardioprotective effects through multiple pathways. Prokineticin 2 (PK2) is a small-molecule secreted protein that plays pivotal parts in cardiomyocyte survival and angiogenesis. However, the role of Met in regulating the PK2 signaling pathway in DCM remains unclear. This experiment explored the effects of Met on high glucose ( $\mathrm{HG}$ )-induced injury through the PK2/PKR pathway in vivo and in vitro. Cardiomyocytes isolated from adult or AKT-knockout mice were treated with HG (33 mmol/L) and PK2 or AKT1/2 kinase inhibitor (AKT inhibitor). Heart contraction properties based on cell shortening were evaluated; these properties included the resting cell length, peak shortening (PS), maximum speed of shortening/relengthening $( \pm \mathrm{dL} / \mathrm{dt}$ ), time to $90 \%$ relengthening (TR9o), and time to peak shortening (TPS). Mice with streptozotocin-induced diabetes were treated with Met to evaluate cardiac function, myocardial structure, and the PK2/PKR and AKT/GSK3 $\beta$ pathways. Moreover, H9c2 cardiomyocytes were exposed to $H G$ in the absence or presence of Met with or without the PK2 antagonist PKRA7 or the AKT inhibitor, and apoptotic proteins such as Bax and Bcl-2 and the PK2/PKR and AKT/GSK3 $\beta$ pathways were evaluated using western blot analysis. The prolongation of TRgo and decreases in PS and $\pm \mathrm{dL} / \mathrm{dt}$ caused by HG were ameliorated by PK2 in cardiomyocytes, but the effects of PK2 were ameliorated or negated by the AKT inhibitor and in AKT-knockout mice. Diabetic mice showed metabolic abnormalities, aberrant myocardial enzyme levels, declines in myocardial systolic and diastolic function associated with myocardial fibrosis, and pronounced apoptosis, but these effects were greatly rescued by Met treatment. Moreover, PK2, PKR1, and PKR2 expression and p-AKT/AKT and p-GSK3 $\beta / G S K 3 \beta$ ratios were decreased in diabetic mice, and these decreases were attenuated by Met. Likewise, H9c2 cells exposed to HG showed reduced PK2/PKR expression and decreased $p-A K T / A K T$ and $p$-GSK3 $\beta / G S K 3 \beta$ ratios, and these effects were nullified by Met. In addition, the effects of Met on cardiomyocytes exposed to HG were abolished 
after intervention with PKRA7 or the AKT inhibitor. These results suggest that Met can activate the PK2/PKR-mediated AKT/GSK3 $\beta$ pathway, thus improving cardiac function and alleviating apoptosis in DM mice.

Keywords: diabetic cardiomyopathy, prokineticin 2, prokineticin 2 receptors, AKT/GSK3 $\beta$ signaling pathway, apoptosis

\section{INTRODUCTION}

Diabetes mellitus (DM) is a metabolic disease caused by a combination of genetic and environmental factors that is characterized by persistent hyperglycemia and insulin deficiency. Currently, the diabetic population is becoming younger, and the International Diabetes Federation has reported that the total number of people with diabetes is expected to reach 642 million by 2040 (von Scholten et al., 2015; Ogurtsova et al., 2017). Diabetes is a strong risk factor for cardiovascular morbidity and mortality (Agrawal et al., 2006). Nowadays, the combinations of traditional Chinese medicines and modern Western medicine are widely used in clinical treatment to prevent or ameliorate the development of diabetic complications (Ceylan Isik et al., 2008). Diabetic cardiomyopathy (DCM) is a structural and functional disorder of the heart caused by diabetes (Rubler et al., 1972). Alterations in mitochondrial, apoptotic, intracellular $\mathrm{Ca}^{2+}$ anomalies, oxidative stress, as well as the expression of $\mathrm{p}-\mathrm{AKT}$, GSK3 $\beta$ all lead to damaged myocardial structure and function in diabetics (Wold et al., 2005; Zhang et al., 2012). In the current work, the specific or tissue selective PI3K modulators and AKT activation were considered as the potential treatment targets of DCM (Bi et al., 2020). However, the mechanisms underlying DCM remain unclear. Therefore, clinical studies are urgently needed to explore these mechanisms.

Prokineticin 2 (PK2) is an $8-\mathrm{kDa}$ small-molecule secreted protein (Mollay et al., 1999). PK2 belongs to the prokineticin protein family, which participates in many biological processes, such as angiogenesis, hematopoiesis, and inflammation, by activating downstream signaling pathways (Martin et al., 2011; Maftei et al., 2014; Kurebayashi et al., 2015; Arora et al., 2016; Gordon et al., 2016). PK2 participates in myocardial diseases by binding to two agonist receptors, prokineticin receptor 1 (PKR1) and prokineticin receptor 2 (PKR2) (Negri et al., 2009). Insulin resistance, cardiac lipid deposition, interstitial fibrosis, and myocardial systolic diastolic function have been observed in PKR1-knockout mice (Boulberdaa et al., 2011a; Dormishian et al., 2013). In addition, AKT plays crucial roles in cardiac growth, coronary angiogenesis, and metabolic regulation (De Los Santos et al., 2017; Qi et al., 2017; Landa-Galvan et al., 2020). Recently, Su et al. (2020) demonstrated that PK2 relieves hypoxia/reoxygenation-induced injury in $\mathrm{H} 9 \mathrm{c} 2$ cardiomyocytes by activating the AKT pathway. In our recent study, the role of the PK2-mediated AKT/GSK3 $\beta$ pathway in the development of DCM was investigated.

Metformin (Met) is a well-known therapeutic drug for diabetes. Met reduces blood glucose mainly by activating AMPactivated protein kinase (AMPK) and non-AMPK pathways. Met is thought to exert its primary antidiabetic action through the suppression of hepatic glucose production (Foretz et al., 2019). Met is also used in the treatment of diabetes complications such as DCM. Metformin ameliorates metabolic disorders, reduces ROS production and cardiac cell death, and improves myocardial structure and function on DCM (Sanit et al., 2019; Yang et al., 2019). Met also protects against brain and liver injury by activating AKT phosphorylation (Naicker et al., 2016; Xu et al., 2016). However, whether Met exerts a positive effect on DCM by regulating the AKT/GSK3 $\beta$ signaling pathway via PK2 is still unclear. Therefore, this study was conducted to elucidate the effects and the possible mechanisms of action of Met in high glucose (HG)-treated cardiomyocytes and DM mice myocardium.

\section{MATERIALS AND METHODS}

\section{Animals}

Male C57BL/6J mice (22 \pm 2 g, 5-6 weeks of age) were purchased from Jinan Pengyue Experimental Animal Breeding Co., Ltd. (China). AKT-knockout mice were provided by the University of Wyoming (Laramie, WY, United States). The animal protocols were in accordance with the Guide for the Care and Use of Laboratory Animals, and the use of the animals was approved by the Institutional Animal Care and Use Committee at Hubei University of Science and Technology.

\section{Cardiomyocyte Isolation and Mechanical Analyses}

Hearts from adult wild-type or AKT-knockout mice were immediately perfused and then digested for $15 \mathrm{~min}$. Then, the left ventricles were removed, and $\mathrm{Ca}^{2+}$ was added to a concentration of $1.25 \mathrm{mmol} / \mathrm{L}$. The cardiomyocytes were used for shortening experiments. The mechanical properties of myocytes were evaluated with an IonOptix SoftEdge system. The resting cell length, time to peak shortening (TPS), peak shortening (PS), time to $90 \%$ relengthening $\left(\mathrm{TR}_{90}\right)$, and maximum speed of shortening/relengthening $( \pm \mathrm{dL} / \mathrm{dt})$ were measured to evaluate cell shortening and relengthening after stimulation at $0.5 \mathrm{~Hz}$ (Aberle et al., 2004). The cells were incubated with KrebsHenseleit bicarbonate solution containing HG (33 $\mathrm{mmol} / \mathrm{L})$ with or without PK2 (10 nmol/L, Sigma) for $4 \mathrm{~h}$ to observe the effect of PK2 on cardiac functional impairment induced by HG.

\section{Experimental DM Model Establishment and Met Treatment}

Mice were randomized into three groups: a control group $(n=20)$, a DM group $(n=25)$, and a DM-Met group $(n=25)$. 


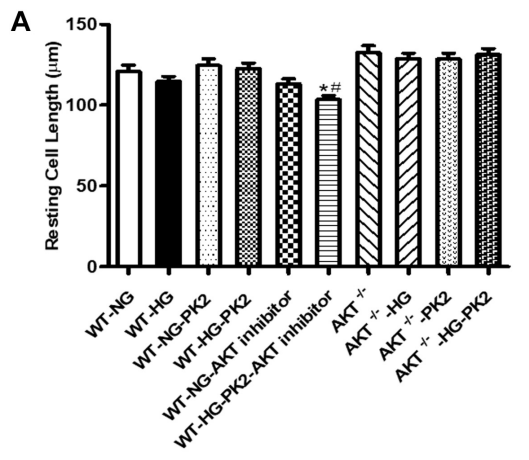

D

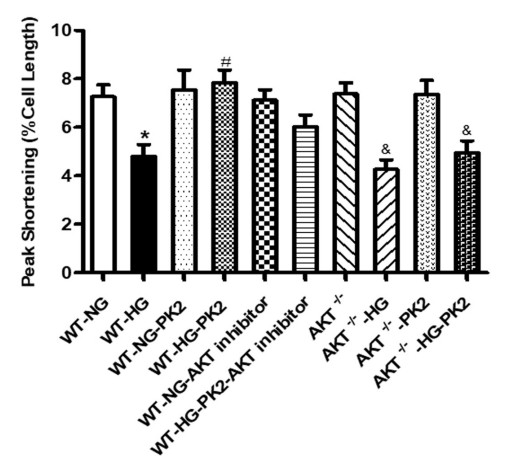

B

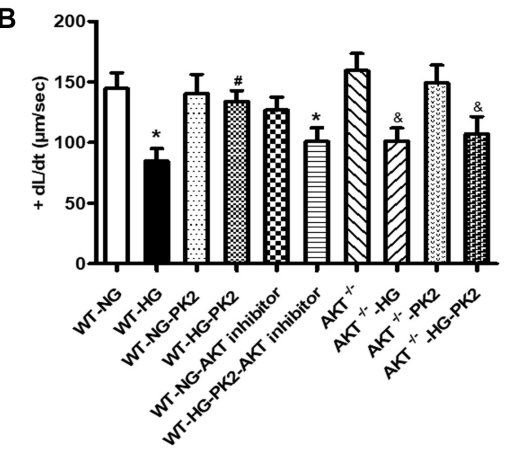

E

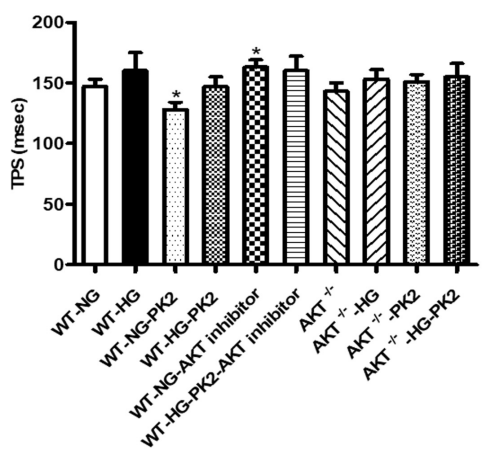

C

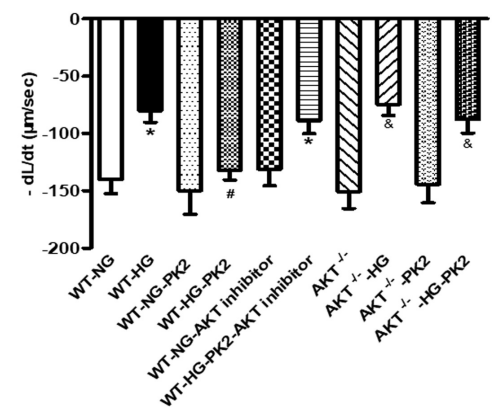

$\mathbf{F}$

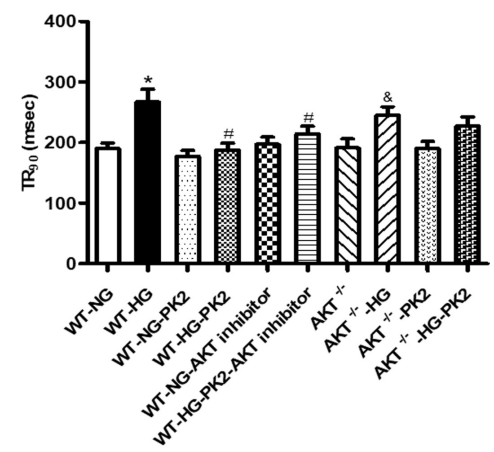

FIGURE 1 | Effect of PK2 on cardiomyocyte shortening. WT, wild-type mouse cardiomyocytes; AKT-/-, AKT-knockout mouse cardiomyocytes; NG, normal glucose; HG, high glucose; NG-PK2, normal glucose plus PK2; HG-PK2, high glucose plus PK2. (A) Resting cell length. (B) +dL/dt. (C) -dL/dt. (D) PS. (E) TPS. (F) TR90. The data are mean \pm SEM; ${ }^{*} P$ value less than 0.05 versus the WT-NG; ${ }^{P} P$ value less than 0.05 versus the WT-HG; ${ }^{\text {P }} \mathrm{P}$ value less than 0.05 versus the $\mathrm{AKT}^{-/}$- group; $n=35-75$ cells per group.

The mice in the DM group and the DM-Met group were injected intraperitoneally with $50 \mathrm{mg} / \mathrm{kg} /$ day streptozotocin (STZ, Sigma, United States) for five consecutive days. The control mice were administered with citrate buffer. Mice with blood glucose levels higher than $16.7 \mathrm{mmol} / \mathrm{L}$ were considered diabetic. The mice in the DM-Met group were given $250 \mathrm{mg} / \mathrm{kg} /$ day Met (SinoAmerican Shanghai Squibb Pharmaceuticals Ltd., China) in drinking water for 16 weeks.

\section{Cell Culture and Treatment}

The H9c2 cell line was purchased from the China Center for Type Culture Collection (CCTCC, China), and the cells were cultured and maintained in Dulbecco's modified Eagle's medium (DMEM, HyClone, United States) supplemented with fetal bovine serum (FBS, 10\%, Gibco, United States), penicillin (1\%, Gibco), and streptomycin at $37^{\circ} \mathrm{C}$ in a humidified atmosphere $\left(5 \% \mathrm{CO}_{2}\right.$ and 95\% air). H9c2 cells were incubated with normal glucose (NG, $5.5 \mathrm{mmol} / \mathrm{L})$ or $\mathrm{HG}(33 \mathrm{mmol} / \mathrm{L})$ for $72 \mathrm{~h}$ with or without Met (2.5 mmol/L, Meilunbio, China), a PK2 antagonist (PKRA7, $10 \mu \mathrm{mol} / \mathrm{L}$, Sigma), and an AKT1/2 kinase inhibitor (AKT inhibitor, $10 \mu \mathrm{mol} / \mathrm{L}$, Sigma).

\section{Echocardiographic Assessment}

Mice were anesthetized with isoflurane. Each rat was fixed on a rat plate, and the chest area was shaved. Cardiac function was evaluated using a two-dimensional guided M-mode echocardiography. Basic hemodynamic parameters, such as the heart rate (HR), left ventricular ejection fraction (LVEF), left ventricular fractional shortening (LVFS), left ventricular posterior wall thickness in diastole (LVPWD), and left ventricular internal dimension in end-diastole (LVIDD), were measured and calculated.

\section{Histopathologic Analysis}

Cardiac tissue was fixed in $4 \%$ paraformaldehyde for $24 \mathrm{~h}$ and then embedded and sectioned. The sections were stained with hematoxylin and eosin (HE) and Masson's trichrome. Myocardial microstructure was observed under a light microscope. Additional cardiac tissue was fixed in electron microscopy fixative and embedded in an acetone-812 embedding agent. The tissues were cut into 60-nm-thick sections and stained with uranyl acetate and lead citrate. Myocardial ultramicrostructure was observed by electron microscopy.

\section{Biochemical Indicators}

Blood was collected from each group of animals, stored at $4^{\circ} \mathrm{C}$ overnight, and centrifuged at $3000 \mathrm{r} / \mathrm{min}$ for $15 \mathrm{~min}$ at $4^{\circ} \mathrm{C}$ in a low-temperature ultracentrifuge. The levels of lactic dehydrogenase (LDH), aspartate aminotransferase (AST), creatine kinase (CK), total cholesterol (TC), and triglyceride 

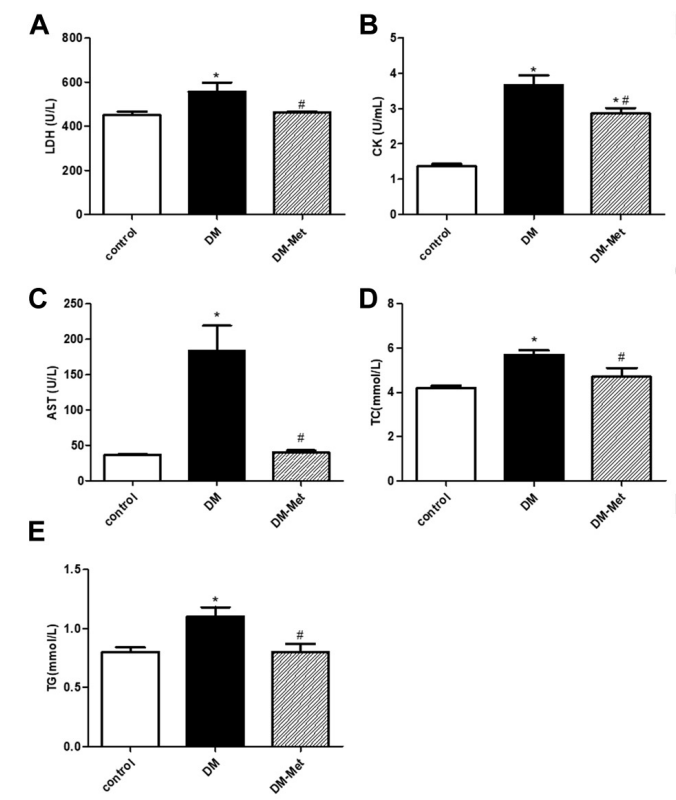

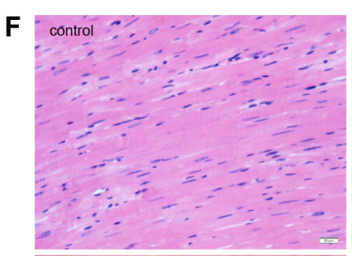

G

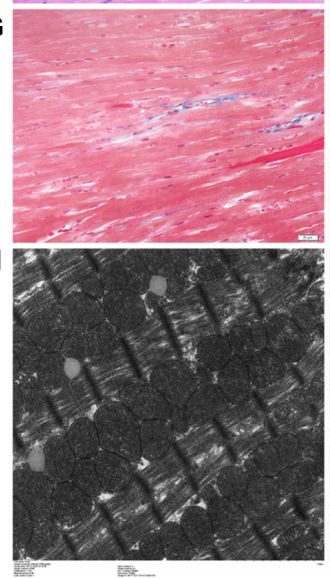

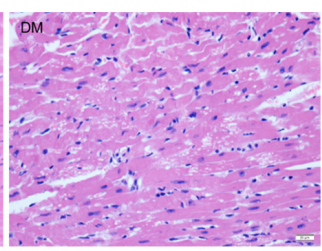
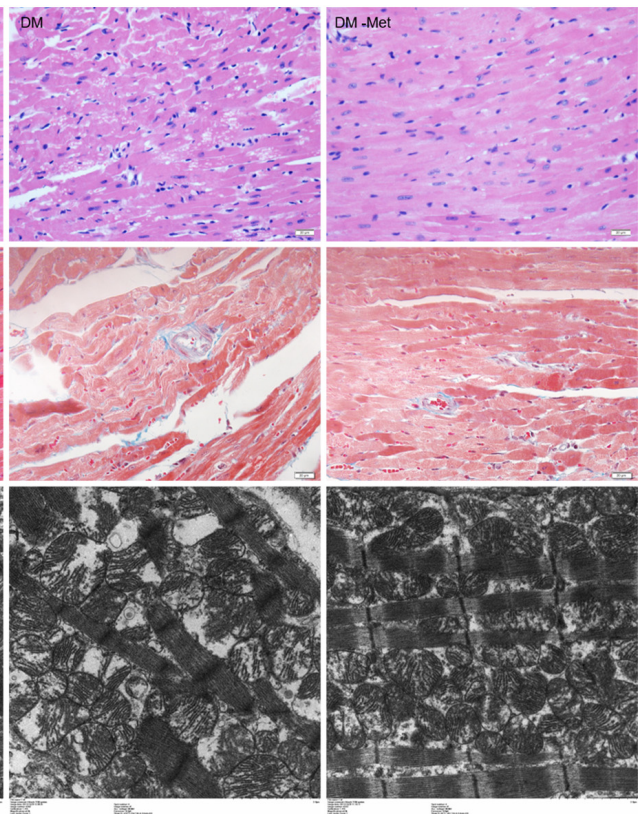

FIGURE 2 | Met protected against myocardial injury in DM mice. (A) LDH, (B) CK, (C) AST, (D) TC, and (E) TG levels. $n=8-22$ per group. (F) HE staining of cardiomyopathy tissue. (G) Masson's trichrome staining of cardiomyopathy tissue. (H) Transmission electron microscopy images of the left ventricle. The data are mean \pm SEM; * $P$ value less than 0.05 versus the control group; ${ }^{*} P$ value less than 0.05 versus the DM group; magnification $=400 \times$; scale bar $=20 \mu \mathrm{m} ; n=5$ per group.

TABLE 1 | Primer sequences.

\begin{tabular}{lll}
\hline Primers & Forward sequence & Reverse sequence \\
\hline PKR1 & 5'-GGCTTCCAGACAGAGCAG & 5'-GACAGTCACAAAGCAGA \\
& ATC-3' & GCGTA-3' \\
PKR2 & 5'-CTACTTCCTCTTCGTCTT & 5'-AGAAGTCTCGCACTATGG \\
& CGGG-3' & TAAAGC-3' \\
\hline
\end{tabular}

(TG) in the serum were tested according to procedures from the Nanjing Jiancheng Institute of Biological Engineering (China).

\section{Real-Time Quantitative Fluorescence PCR Analysis}

Total RNA was extracted from heart tissues with TRIzol reagent. Reverse transcription was performed to synthesize cDNA. The samples were incubated at $65^{\circ} \mathrm{C}$ for $5 \mathrm{~min}$ and then cooled on ice. Reaction buffer $(5 \times)$, mixed dNTPs, RiboLock RNase inhibitor, and RevertAid $\mathrm{M}-\mathrm{MuLV}$ reverse transcriptase were sequentially added. The samples were incubated at $42^{\circ} \mathrm{C}$ for $60 \mathrm{~min}$ in a PCR instrument, and the reverse transcriptase was inactivated at $70^{\circ} \mathrm{C}$ for $5 \mathrm{~min}$. The PCR amplification reaction conditions were as follows: $95^{\circ} \mathrm{C}$ for $10 \mathrm{~min}, 95^{\circ} \mathrm{C}$ for $15 \mathrm{~s}$, and annealing at $60^{\circ} \mathrm{C}$ for $60 \mathrm{~s}$ for 40 cycles. Melting curve analysis was performed from $60^{\circ} \mathrm{C}$ to $95^{\circ} \mathrm{C}$ in $0.3^{\circ} \mathrm{C}$ increments over $15 \mathrm{~s}$. The results were analyzed by the $\Delta \Delta \mathrm{CT}$ method. Table 1 shows the primer sequences used for the PCR experiment.

\section{Immunohistochemistry}

After dewaxing and hydration of the conventionally prepared paraffin slices, the samples were incubated with primary antibodies (working concentration: $1: 1000$ ) at $4^{\circ} \mathrm{C}$ for $12 \mathrm{~h}$ and then incubated with secondary antibodies for $1 \mathrm{~h}$. A solution of diaminobenzidine was added dropwise, and the slices were observed under a microscope. The procedure was carried out according to the kit instructions.

\section{TUNEL Assay}

Apoptosis was tested with a terminal deoxynucleotidyl transferase-mediated dUTP-biotin nick end labeling (TUNEL) assay in paraffin-embedded heart sections. Ten fields were analyzed for each slice, and the number of positive cells among cardiomyocytes was determined for each field. The apoptosis rates were calculated as the percentages of positive cells (Long et al., 2018).

\section{Western Blot Analysis}

Fifty milligrams of myocardial tissue was weighed, and RIPA lysis buffer was added. A BCA Protein Assay Kit (Beyotime, China) was used to test the protein concentration. Protein (50 mg) was added to each lane of a 12\% SDS-polyacrylamide gel, and the proteins were separated and transferred to a polyvinylidene fluoride (PVDF) membrane. The membrane was blocked and incubated overnight at $4^{\circ} \mathrm{C}$ with AKT, p-AKT, GSK3 $\beta$, p-GSK3 $\beta$, Bax, Bcl-2 (1:1000, Cell Signaling Technology, United States), PK2 (1:1000, Abcam, United States), PKR1, and PKR2 (1:2000, Santa Cruz) antibodies. After incubation with 

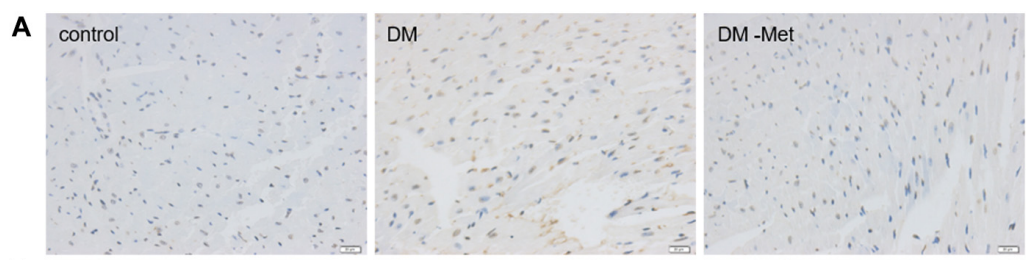

B
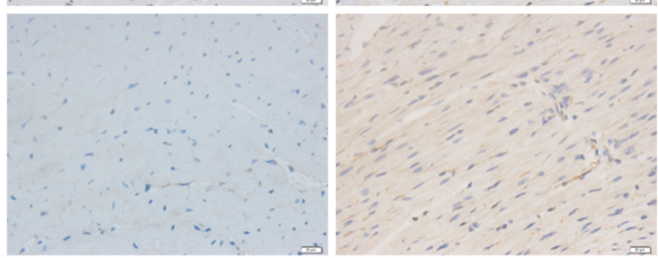

C
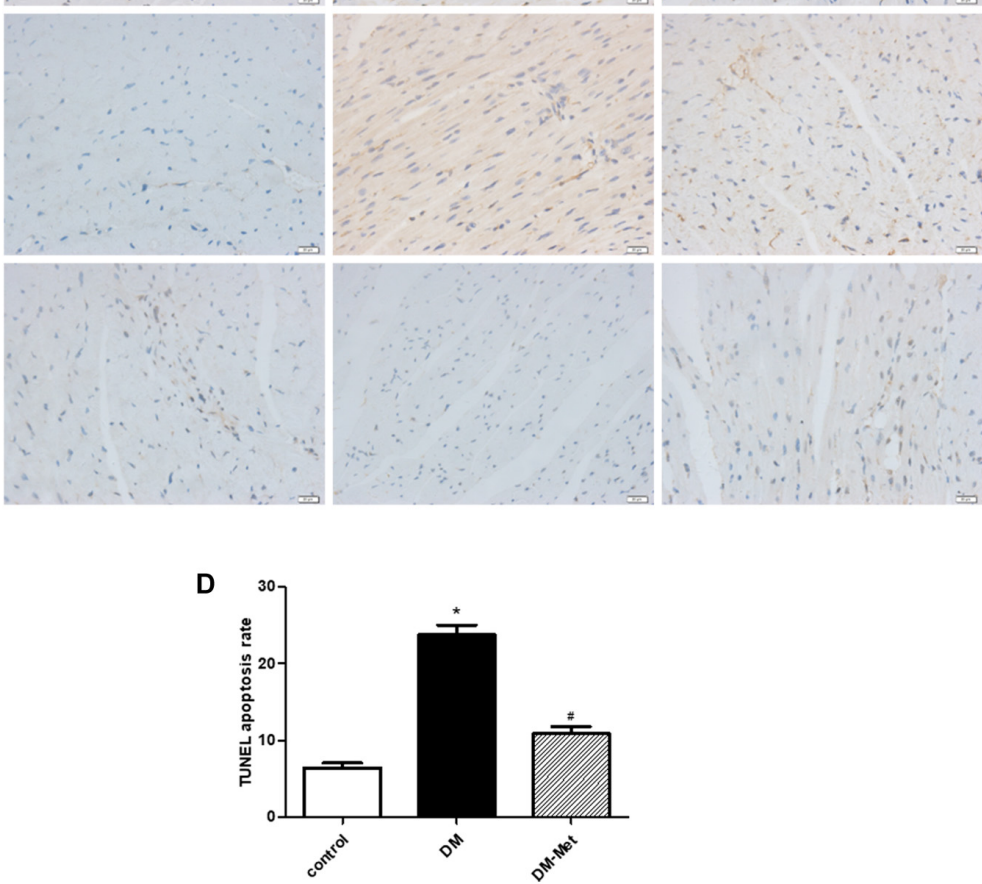

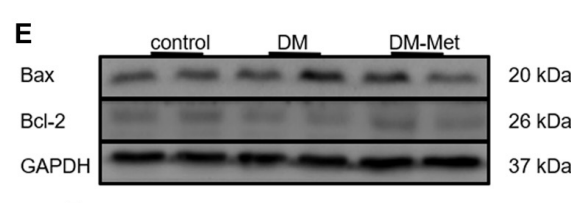

$\mathbf{F}$
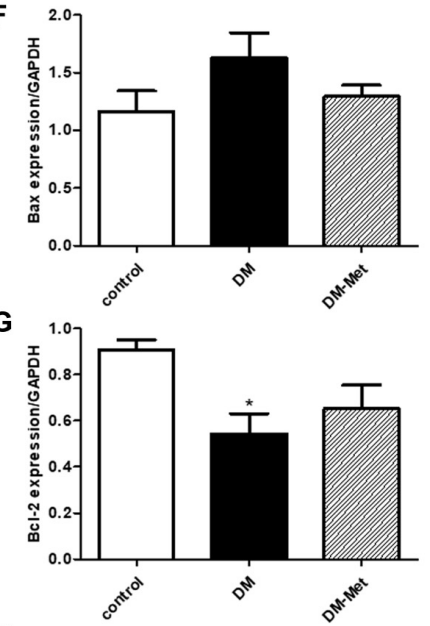

$\mathbf{H}$

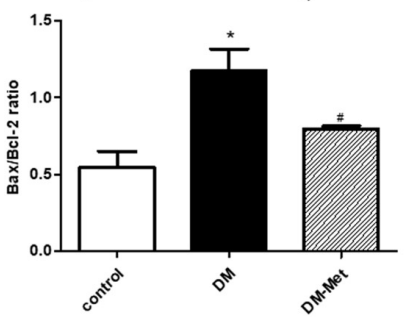

FIGURE 3 | Met prevented diabetes-induced cardiomyocyte apoptosis. (A) TUNEL staining. (B) Immunohistochemical staining for Bax. (C) Immunohistochemical staining for Bcl-2. (D) Analysis of TUNEL-positive cells. (E) Images of Bcl-2 and Bax protein levels. (F) Analysis of Bax. (G) Analysis of Bcl-2. (H) Analysis of the $\mathrm{Bax} / \mathrm{Bcl}-2$ ratio. The data are mean $\pm \mathrm{SEM} ;{ }^{*} P$ value less than 0.05 versus the control group; ${ }^{\#} P$ value less than 0.05 versus the DM group; $n=3-5$ per group.

secondary antibodies for an hour, the analysis was completed using an Enhanced Chemiluminescence Kit (Meilunbio, China).

\section{Statistical Analysis}

The data are presented as the mean \pm standard error of the mean (SEM). T-tests or one-way ANOVA was performed for all statistical analyses. $T$-tests were applied to analyze differences between two groups, and one-way ANOVA (Student-NewmanKeuls) was used to analyze differences among three or more groups. A $P$ value less than 0.05 was considered to indicate statistical significance.

\section{RESULTS}

\section{Effect of PK2 on Cardiomyocyte Shortening}

As shown in Figure 1, compared with NG exposure, HG (33 mmol/L) exposure decreased the $\pm \mathrm{dL} / \mathrm{dt}$ and PS and prolonged the $\mathrm{TR}_{90}$ significantly but did not affect the resting cell length or TPS. PK2 significantly alleviated the HG-induced abnormalities in PS, $\pm \mathrm{dL} / \mathrm{dt}$, and $\mathrm{TR}_{90}$ but had no effect on TPS or resting cell length. The effects were ameliorated or negated in AKT-knockout mouse cardiomyocytes and by AKT inhibitor treatment. These data strongly indicate that PK2 plays a pivotal role in the diabetic heart and that the important roles of AKT in $\mathrm{PK} 2$ provide benefits against glucose toxicity.

\section{Met Improved General Features and Echocardiographic Properties in DM Mice}

Body weight (BW), heart weight (HW), and blood glucose levels reflect the general health state of mice. The results showed that BW and HW were significantly decreased and that the heartto-body weight ratio (HW/BW) was significantly increased in the DM group compared with the control group; these changes were overtly reversed by Met treatment (Table 2 ). In addition, diabetic mice displayed significantly higher blood glucose levels than control mice. Treatment with Met improved the blood glucose levels in diabetic mice but did not restore them to normal levels. We have published the data of BW and blood glucose (Liu et al., 2019).

Echocardiography is a technique for examining the anatomy and function of the heart. Echocardiographic evaluation revealed 
A

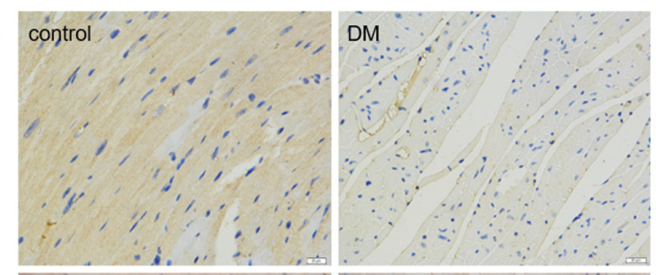

B

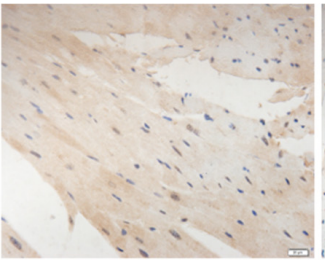

C

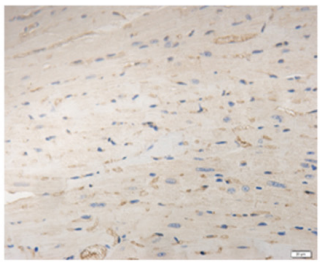

D

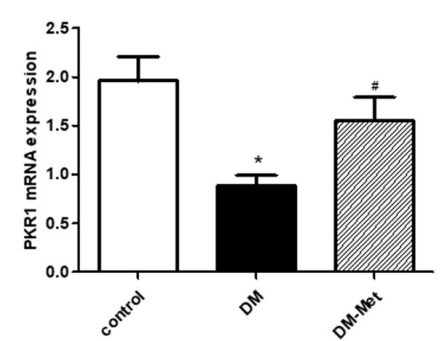

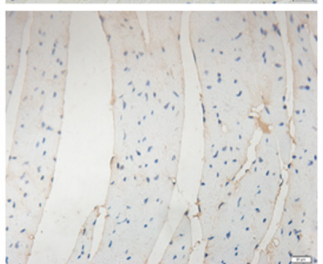
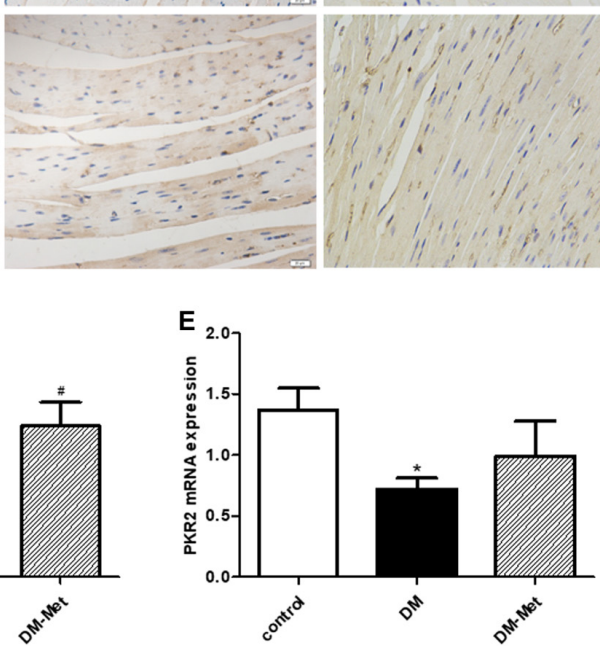
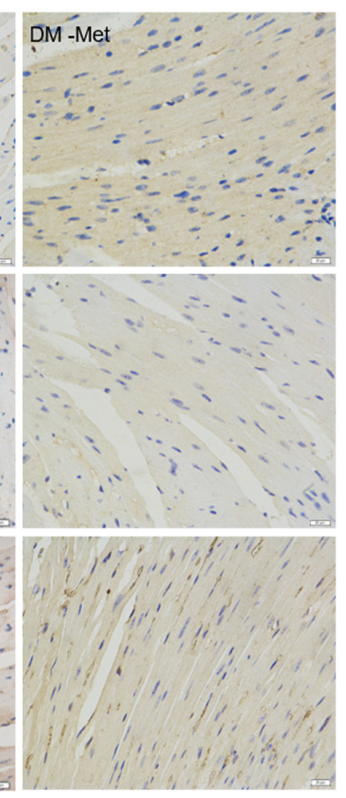

.

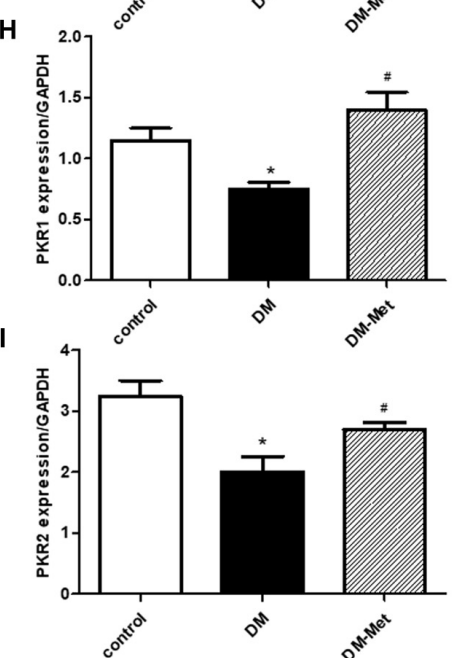

FIGURE 4 | Met increased the expression of PK2/PKR signaling pathway members in DM mice. (A) Immunohistochemical staining for PK2. (B) Immunohistochemical staining for PKR1. (C) Immunohistochemical staining for PKR2. $n=4-5$ per group. (D) Expression of PKR1 mRNA, as determined by RT-PCR. (E) Expression of PKR2 mRNA, as determined by RT-PCR. (F) Images of PK2, PKR1, and PKR2 protein levels. (G) Analysis of PK2. (H) Analysis of PKR1. (I) Analysis of PKR2. The data are mean \pm SEM; * $P$ value less than 0.05 versus the control group; ${ }^{*} P$ value less than 0.05 versus the DM group; $n=4-8$ per group.

that LVPWD and LVIDD were slightly increased in DM mice, but these increases were mitigated by Met. Moreover, HR was significantly reduced in DM mice, and the reduction was partly eliminated by Met. Moreover, DM mice showed remarkable reductions in both LVEF and LVFS that were notably nullified by Met (Table 2).

\section{Met Protected Against Myocardial Injury in DM Mice}

Myocardial zymograms are recognized as effective indicators of cardiac function. The levels of $\mathrm{LDH}, \mathrm{CK}$, and AST in serum were significantly increased in the DM group compared to the control group, the effects of which were reversed by Met administration (Figures 2A-C). Moreover, TC and TG levels were significantly elevated in DM mice, indicating that DM mice had abnormal blood lipid metabolism, the effects of which were ameliorated by Met (Figures 2D,E).

Hematoxylin and eosin staining showed that the myocardial cells in the control group were clearly visible and arranged neatly, while those in the DM group showed disordered
TABLE 2 | Met improved general features and echocardiographic properties in DM mice.

\begin{tabular}{lccc}
\hline Parameter & control & DM & DM-Met \\
\hline HW $(\mathrm{mg})$ & $144.9 \pm 2.8$ & $99.9 \pm 6.3^{\star}$ & $111.6 \pm 4.9^{*}$ \\
HW/BW $(\mathrm{mg} / \mathrm{g})$ & $4.6 \pm 0.1$ & $5.2 \pm 0.2^{*}$ & $4.0 \pm 0.2^{\star \#}$ \\
HR $(\mathrm{bpm})$ & $505.21 \pm 36.84$ & $372.71 \pm 34.22^{\star}$ & $462.25 \pm 6.24$ \\
LVPWD (mm) & $0.68 \pm 0.06$ & $0.72 \pm 0.04$ & $0.58 \pm 0.01$ \\
LVIDD (mm) & $3.38 \pm 0.12$ & $3.57 \pm 0.06$ & $3.43 \pm 0.05$ \\
LVEF (\%) & $72.13 \pm 3.17$ & $58.31 \pm 4.18^{\star}$ & $71.32 \pm 2.64^{\#}$ \\
LVFS $(\%)$ & $40.64 \pm 2.66$ & $30.37 \pm 2.75^{\star}$ & $39.72 \pm 2.24^{\#}$
\end{tabular}

HR, heart rate; LVPWD, left ventricular posterior wall thickness; LVIDD, left ventricular internal dimension in end-diastole; LVEF, left ventricular ejection fraction; $L V F S$, left ventricular fractional shortening. The data are mean \pm SEM; ${ }^{*} P$ value less than 0.05 versus the control group; " $P$ value less than 0.05 versus the DM group; $n=4-11$ per group.

myocardial fiber arrangement and vacuolization. Interestingly, the myocardial fibers were denser in the Met treatment group than in the DM group, and less vacuolization was observed (Figure 2F). 


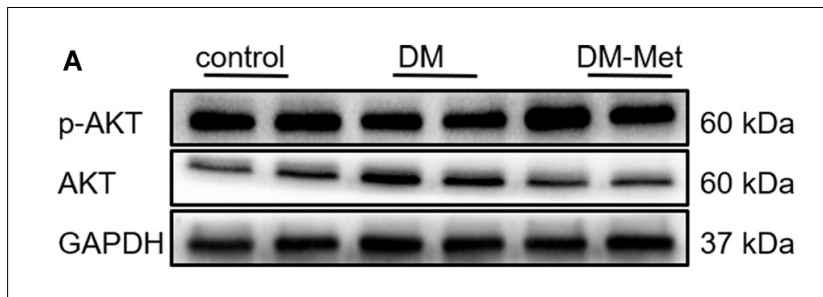

B

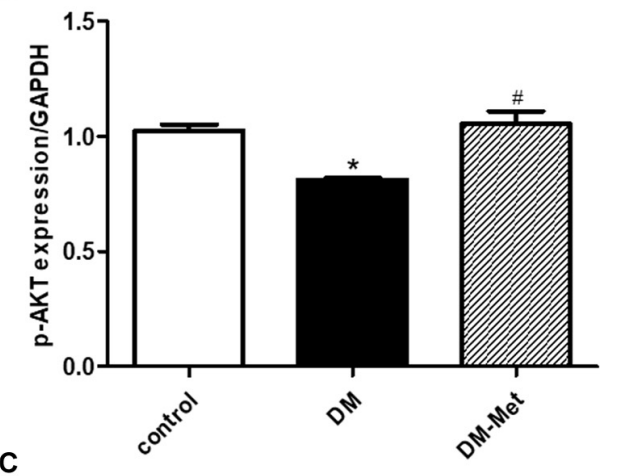

C
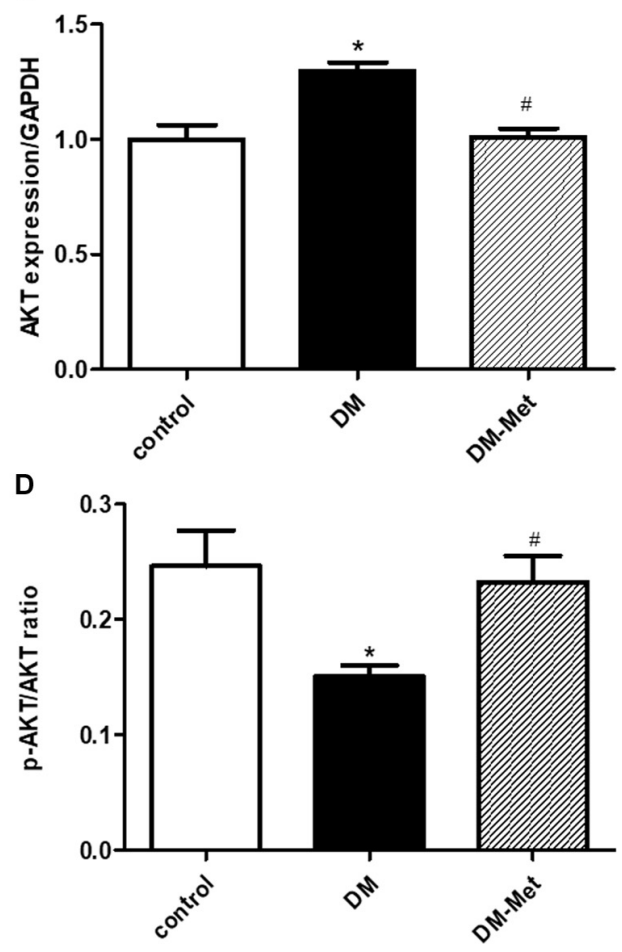

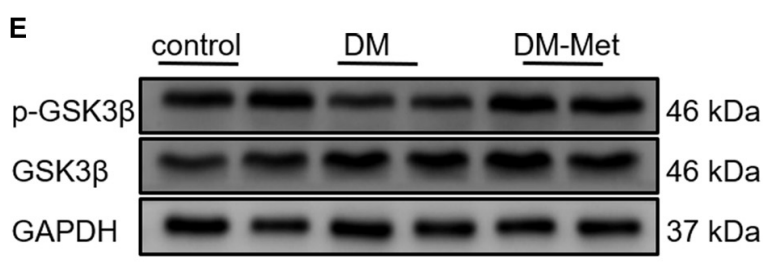

$\mathbf{F}$
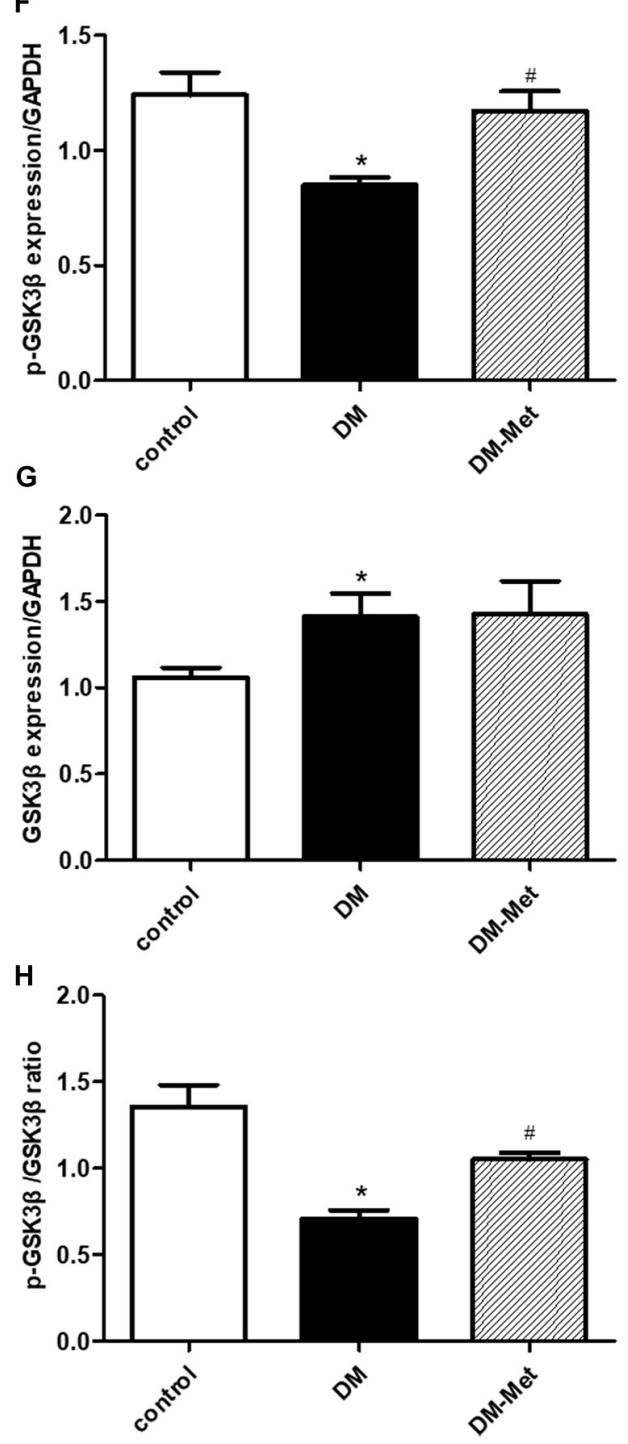

FIGURE 5 | Met activated the AKT/GSK3 $\beta$ signaling pathway in DM mice. (A) Images of p-AKT and AKT protein levels. (B) Analysis of $p$-AKT. (C) Analysis of AKT. (D) Analysis of the p-AKT/AKT ratio. (E) Images of p-GSK3 $\beta$ and GSK3 $\beta$ protein levels. (F) Analysis of p-GSK3 $\beta$. (G) Analysis of GSK3 $\beta$. (H) Analysis of the p-GSK3 $\beta$ /GSK3 $\beta$ ratio. The data are mean \pm SEM; ${ }^{*} P$ value less than 0.05 versus the control group; ${ }^{*} P$ value less than 0.05 versus the DM group; $n=4$ per group.

Masson's trichrome staining showed that there was less blue collagen in the myocardial interstitium in the control group than in the other groups. In contrast, increased interstitial fibrosis was evident in the DM mice hearts, and the fibrotic changes were significantly attenuated after Met administration (Figure 2G).

Electron microscopy revealed that in the control group, the mitochondria were intact, the myofilaments were neat and complete, and the Z-line and M-line were clear. However, in the DM group, the myocardial cells were arranged in a disorderly manner, the mitochondria were swollen, and myofilament fracture was present. Met ameliorated these abnormalities, causing the myofilament arrangement to be more regular and the mitochondrial structure to be more complete and reducing myofilament fracture (Figure $2 \mathbf{H}$ ). 

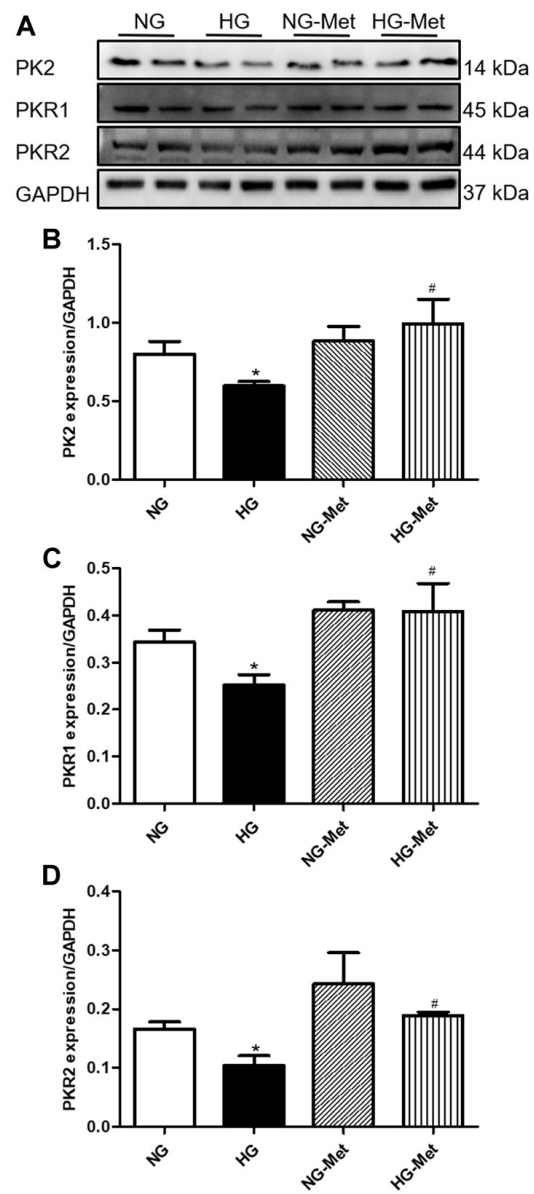

E

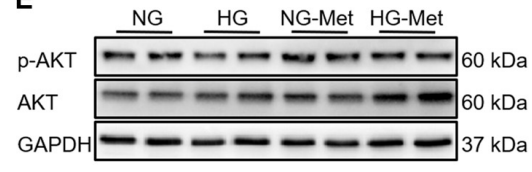

F

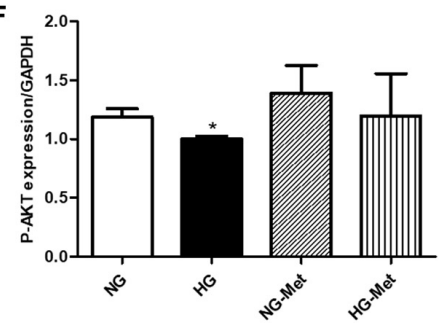

G

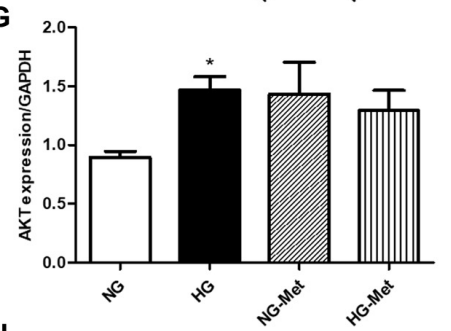

H

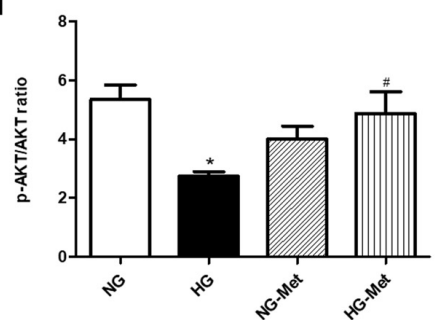

I

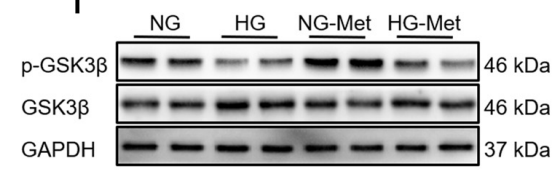

J

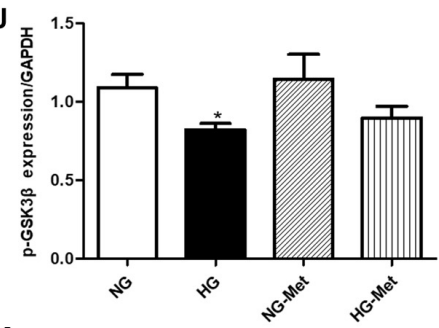

K

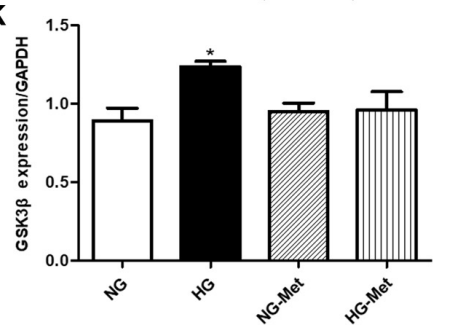

$\mathbf{L}$

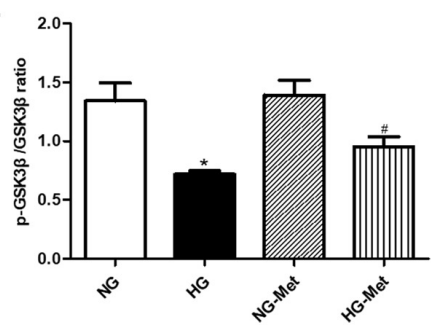

FIGURE 6 | Effects of Met on the PK2/PKR and AKT/GSK3 $\beta$ signaling pathways in HG-treated cardiomyocytes. NG-Met, normal glucose plus metformin; HG-Met, high glucose plus metformin. (A) Images of PK2, PKR1, and PKR2 protein levels. (B) Analysis of PK2. (C) Analysis of PKR1. (D) Analysis of PKR2. (E) Images of p-AKT and AKT protein levels. (F) Analysis of p-AKT. (G) Analysis of AKT. (H) Analysis of the p-AKT/AKT ratio. (I) Images of $p-G S K 3 \beta$ and GSK3 $\beta$ protein levels. (J) Analysis of $p$-GSK3 $\beta$. (K) Analysis of GSK3 $\beta$. (L) Analysis of the $p$-GSK3 $\beta / G S K 3 \beta$ ratio. The data are mean \pm SEM; ${ }^{*} P$ value less than 0.05 versus the NG group; \# $P$ value less than 0.05 versus the HG group; $n=3-4$ per group.

\section{Met Prevented Diabetes-Induced Cardiomyocyte Apoptosis}

TUNEL staining was used to detect apoptotic cardiomyocytes. The data showed that there were many more apoptotic cardiomyocytes (stained in brown) in the DM group than in the control group, but Met treatment significantly decreased DM-induced myocardial cell apoptosis (Figures 3A,D).

Apoptosis-related proteins play important roles in apoptosis progression. We thus carried out a more in-depth experiment to investigate whether apoptosis-related proteins affected the amelioration of DM-induced cardiomyocyte apoptosis by Met. As shown in Figures 3B,C, Bax protein upregulation and Bcl-2 protein downregulation (as assessed using immunohistochemical staining) were evident in the DM group compared with the control group, and these effects were reversed by Met. Consistent with the immunohistochemical staining results, the western blot results showed that Bax expression was enhanced in the DM group, whereas $\mathrm{Bcl}-2$ expression was remarkably reduced; these changes were partly attenuated by Met administration.
Furthermore, the Bax/Bcl-2 ratio was elevated in the DM group compared to that in the control group, the effect of which was markedly abrogated by Met administration (Figures 3E-H).

\section{Met Increased the Expression of PK2/PKR Signaling Pathway Members in DM Mice}

Previous studies have suggested that PK2 plays essential roles in the survival, proliferation, and apoptosis of cardiomyocytes. To further elucidate the role of PK2 in Met-mediated cardioprotection, we measured the effects of Met on PK2/PKR signaling pathway members. The results of immunohistochemical staining showed that the expression of PK2, PKR1, and PKR2 (brown labeling) was lower in the $\mathrm{DM}$ group than in the control group, the effects of which were ablated by Met treatment (Figures 4A-C). Constant to immunohistochemical results, the levels of PKR1 and PKR2 mRNA were also significantly downregulated, the effects of which were partly or considerably reversed by Met treatment 

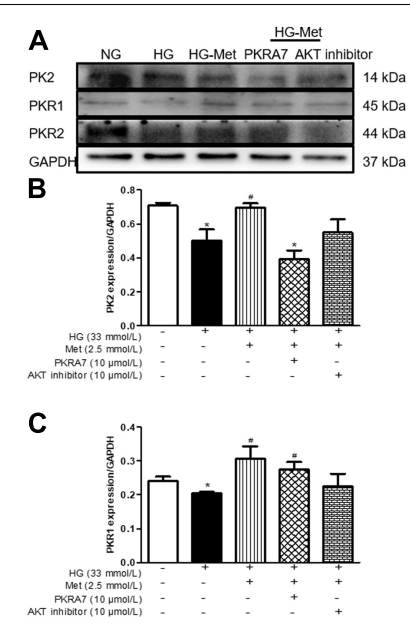

D

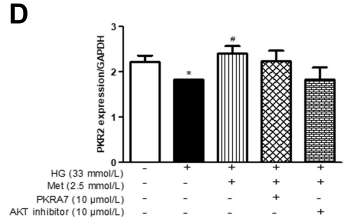

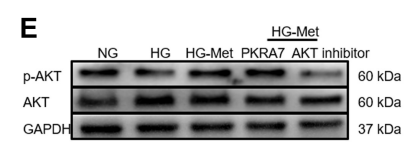

$\mathbf{F}$

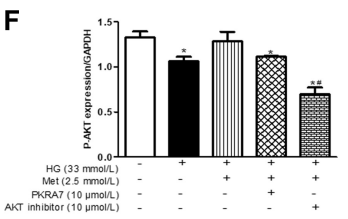

G

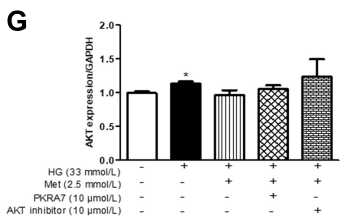

H

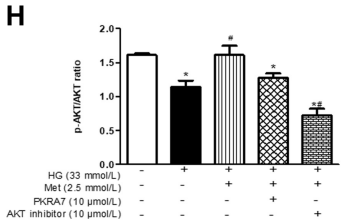

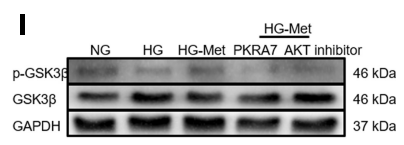

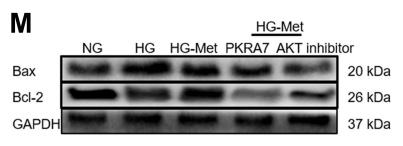

J

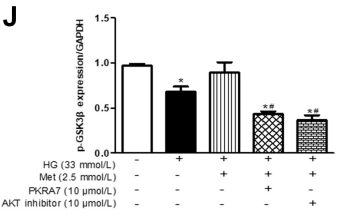

$\mathbf{N}$
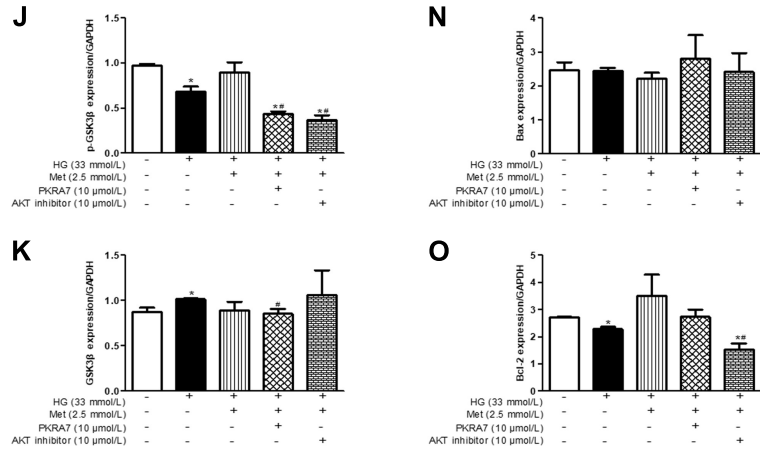

L

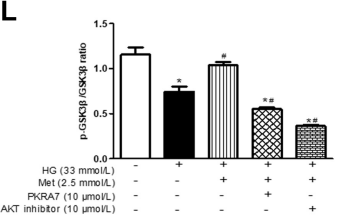

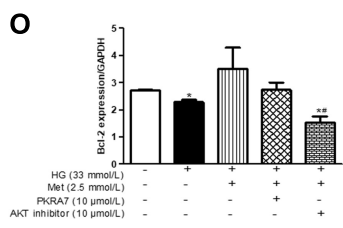

$\mathbf{P}$

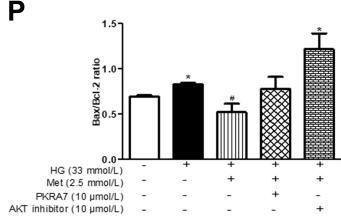

FIGURE 7 | A PK2 antagonist and an AKT inhibitor blocked the effects of Met on HG-treated cardiomyocytes. (A) Images of PK2, PKR1, and PKR2 protein levels. (B) Analysis of PK2. (C) Analysis of PKR1. (D) Analysis of PKR2. (E) Images of p-AKT and AKT protein levels. (F) Analysis of p-AKT. (G) Analysis of AKT. (H) Analysis of the p-AKT/AKT ratio. (I) Images of $p$-GSK3 $\beta$ and GSK3 $\beta$ protein levels. (J) Analysis of $p$-GSK3 $\beta$. (K) Analysis of GSK3 $\beta$. (L) Analysis of the $p$-GSK3 $\beta / G S K 3 \beta$ ratio. (M) Images of Bcl-2 and Bax protein levels. (N) Analysis of Bax. (O) Analysis of Bcl-2. (P) Analysis of the Bax/Bcl-2 ratio. The data are mean \pm SEM; ${ }^{P} P$ value less than 0.05 versus the NG group; ${ }^{P}$ value less than 0.05 versus the $\mathrm{HG}$ group; $n=3-4$ per group.

(Figures 4D,E). Furthermore, the western blot results showed that the levels of PK2, PKR1, and PKR2 protein were markedly lower in the DM group than in the control group, but higher in the DM-Met group than in the DM group (Figures $4 \mathbf{F}-\mathbf{I}$ ). The data suggest that Met exerts a positive effect against diabetesinduced cardiac injury primarily by increasing the expression of PK2/PKR pathway members.

\section{Met Activated the AKT/GSK3 $\beta$ Signaling Pathway in DM Mice}

Previously obtained evidence has revealed that the cardioprotective actions of $\mathrm{PK} 2 / \mathrm{PKR}$ may involve the phosphorylation of AKT, and impaired AKT activity in response to insulin is a common feature of DM (Wang C.Y. et al., 2016; Su et al., 2020). We therefore assessed the effect of Met on the AKT/GSK3 $\beta$ signaling pathway. As shown in Figure 5, the $\mathrm{p}-\mathrm{AKT} / \mathrm{AKT}$ and $\mathrm{p}-\mathrm{GSK} 3 \beta / \mathrm{GSK} 3 \beta$ ratios were significantly declined in the DM group, the effects of which were notably reversed by Met treatment.

\section{Effects of Met on the PK2/PKR Signaling Pathway in HG-Treated Cardiomyocytes}

To further validate the role of the PK2/PKR pathway in Met-induced cardiomyocyte mechanical responses against HG in vitro, the $\mathrm{PK} 2 / \mathrm{PKR}$ signaling pathway was examined using western blot analysis. The data showed that PK2, PKR1, and PKR2 expression levels were overtly decreased in
$\mathrm{H} 9 \mathrm{c} 2$ cardiomyocytes exposed to $\mathrm{HG}$, but the changes were significantly abrogated by Met treatment (Figures 6A-D).

\section{Met Activated the AKT/GSK3 $\beta$ Signaling Pathway in HG-Treated Cardiomyocytes}

To determine whether Met activates the PK2-mediated AKT/GSK3 $\beta$ signaling pathway in HG-treated cardiomyocytes, $\mathrm{AKT}$ and GSK3 $\beta$ signaling was examined using western blot analysis. As demonstrated in Figures 6E-L, HG incubation markedly decreased the ratio of $\mathrm{p}$-AKT/AKT and $\mathrm{p}-\mathrm{GSK} 3 \beta / \mathrm{GSK} 3 \beta$, the effects of which were negated by Met treatment.

\section{A PK2 Antagonist and an AKT Inhibitor Blocked the Effects of Met on HG-Treated Cardiomyocytes}

To clarify whether PK2 and PK2-mediated AKT play key roles in the Met-induced beneficial response to HG challenge, cardiomyocytes were incubated with NG or HG medium in the absence or presence of Met and PKRA7 or an AKT inhibitor. As shown in Figures 7A-P, incubation with PKRA7 partially or overtly reversed the Met-induced increases in PK2, PKR1, and PKR2 expression. In parallel, the Met-induced elevations in the $\mathrm{p}-\mathrm{AKT} / \mathrm{AKT}$ and $\mathrm{p}-\mathrm{GSK} 3 \beta / \mathrm{GSK} 3 \beta$ ratios were abolished by treatment with PKRA7. In addition, the AKT inhibitor effectively eliminated the effects of Met on the p-AKT/AKT and p-GSK $3 \beta / G S K 3 \beta$ ratios and partially attenuated the effects of 
Met on PK2 and PKR expression. Furthermore, Met failed to regulate the expression of $\mathrm{Bcl}-2$ and Bax when PKRA7 or the AKT inhibitor was applied. These results illustrate that Met exerts protective effects against glucose toxicity through the PK2/PKRmediated AKT/GSK3 $\beta$ pathway.

\section{DISCUSSION}

The salient findings from our current study suggest that the PK2/PKR pathway plays a crucial role in the pathogenesis of $\mathrm{DCM}$ and that Met treatment prevents diabetes-induced glucose and lipid metabolism dysfunction, cardiomyocyte apoptosis, fibrosis, and cardiac insufficiency by stimulating PK2/PKR and the downstream AKT/GSK3 $\beta$ pathway. Glucose toxicity is considered to be the key factor in cardiovascular complications caused by diabetes, but the clinical treatment of DCM is still challenging. Our data reveal a likely role for the PK2/PKR pathway in the anti-DCM effects of Met.

It is well known that myocardial fibrosis and apoptosis contribute to the development of DCM (Gao et al., 2019). Several studies have also shown that left ventricular dysfunction and myocardial apoptosis are closely related, which indicates that inhibition of cardiomyocyte apoptosis can restore cardiac function (Yu et al., 2016). Various apoptotic stimuli, such as hyperglycemia and lipid metabolism dysfunction, can lead to enormous cardiomyocyte loss, which is proven to facilitate contractile dysfunction, fibrosis, and remodeling. Therefore, inhibition of cardiomyocyte fibrosis and apoptosis is thought to be a valid strategy for treating DCM. In the current work, disturbance of glucose and lipid metabolism, overt remodeling mainly presented as disorderly arrangement of myocardial cells, considerable deposition of collagen fibers in the myocardial interstitium, elevated $\mathrm{HW} / \mathrm{BW}$, and compromised cardiac function were observed in diabetic mice. However, longterm administration of Met rescued against DM-induced cardiac remodeling and functional anomalies. Notably, apoptosis-related Bcl-2 family members regulate apoptosis progression. Bax and $\mathrm{Bcl}-2$ are members of the Bcl-2 family, and Bax binds with Bcl-2 to block upstream apoptotic signal transduction and promote cell survival and growth (Thandavarayan et al., 2011; Jafri et al., 2019). We discovered diabetes triggered cardiomyocytes apoptosis and increased $\mathrm{Bax} / \mathrm{Bcl}-2$ ratios in $\mathrm{DM}$ mice compared with control mice. However, Met reduced the diabetes-induced increases in cardiomyocyte apoptosis and the $\mathrm{Bax} / \mathrm{Bcl}-2$ ratio. Thus, it is plausible to speculate that repair of cardiac function upon Met treatment is conducive to the inhibition of cardiomyocyte apoptosis.

Prokineticin 2 expression has been observed in a variety of tissues, such as the brain, heart, and testes. PK2 affects various biological processes, including neuronal survival, olfactory bulb morphogenesis, testis development, and circadian rhythms (Boulberdaa et al., 2011b; Neal et al., 2018). In recent years, evidence has demonstrated that $\mathrm{PK} 2$ protects against oxidative stress in cardiomyocytes and H9c2 cells, suggesting that PK2 is inversely related to apoptosis (Gao et al., 2019). In addition, PK2 treatment can reduce food intake and BW in lean and obesity models (Gardiner et al., 2010). However, the precise functions of PK2 in diabetic-induced cardiac injury have not been determined to date. In this study, we found that PK2 ameliorated HG-induced cardiac contractile dysfunction by elevating PS and $\pm \mathrm{dL} / \mathrm{dt}$ and decreasing $\mathrm{TR}_{90}$ in cardiomyocytes, supporting that PK2 may be the key factor in DCM. Prokineticins exert multiple biological effects by activating PKR1 and PKR2 and mostly differ in their N-terminal sequences. Previous studies have revealed that $\mathrm{PK} 2$ and PKR1 levels are reduced in the late stage of heart failure, but PKR1 overexpression can protect cardiomyocytes from hypoxia injury-elicited apoptosis (Urayama et al., 2007; Gasser et al., 2015). Moreover, accumulating studies have demonstrated that $\mathrm{PK} 2 / \mathrm{PKR}$ is associated with many types of cardiometabolic risk factors and exerts a vital role in cardiovascular disease development (Wang Y. et al., 2016). Upregulation of PK2 manipulates epicardium-derived progenitor cells involved in tissue repair/regeneration in heart diseases, whereas PKR1 knockout in the epicardium results in ventricular hypoplasia, ventricular septal defects, and even embryo necrosis (Curtis et al., 2013). Similarly, PK2 induces the growth of myocardium derived from mice, but the effect is abolished in PKR1-knockout myocardium (Gasser et al., 2015). In addition, PKR1-null mice exhibit cardiomyocyte apoptosis and contractile defects (Boulberdaa et al., 2011a). Recently, numerous studies have explored the cardioprotective effect of Met in the DCM myocardium, but whether Met can activate PK2 and its pathway in DCM remains unclear. Since our data showed that PK2 treatment protected against HG-induced cardiac function compromise in vitro, we made further efforts to investigate the protective effect of Met on HG-induced cardiac damage mediated via regulation of the $\mathrm{PK} 2 / \mathrm{PKR}$ pathway in vitro and in vivo. As expected, our current results suggested that PK2, PKR1, and PKR2 expression was remarkably dampened both in diabetic mouse hearts and $\mathrm{H} 9 \mathrm{c} 2$ cardiomyocytes exposure to HG, which further confirmed that a decrease in PK2 may be the main contributor to myocardial apoptosis in DCM. Interestingly, after administration of Met, PK2, PKR1, and PKR2 expression was significantly increased in vivo and in vitro. In addition, PKRA7 partly or considerably counteracted the beneficial effects of Met against glucose toxicity. Our observations favored a role for activation PK2/PKR pathway in the Met-offered beneficial mechanical effects against HG-induced cardiac damage.

Cumulative evidence suggests that PK2/PKR participates in myocardial survival, angiogenesis, and the hematopoietic system through the AKT and STAT3 signaling pathways (Urayama et al., 2007; Xin et al., 2013). AKT appears to regulate cell survival, growth, apoptosis, and angiogenesis. Abnormal AKT regulation leads to impaired glucose tolerance in diabetic patients (Chen et al., 2001). GSK3 $\beta$, one of the downstream substrates of AKT, is involved in physiological processes including cell death (Wang et al., 2009). It is now recognized that glucose utilization and abnormal free fatty acid oxidation are involved in the development of DCM (Neglia et al., 2007). Thus, activating AKT and inhibiting GSK3 $\beta$ activity can be considered effective treatment strategies for DCM. Our study clearly shows that regulation of the PK2-mediated AKT/GSK3 $\beta$ pathway may be a potential mechanism of the cardioprotective role of Met. 
This possibility is supported by several pieces of experimental data. First, we found that the protective effect of PK2 against HG challenge was completely negated in AKT-knockout mice and AKT inhibitor-treated isolated cardiomyocytes. Second, the results revealed that the $\mathrm{p}$-AKT/AKT and $\mathrm{p}$-GSK3 $\beta / G S K 3 \beta$ ratios were notably decreased not only in diabetic mice but also in HG-challenged $\mathrm{H} 9 \mathrm{c} 2$ cardiomyocytes, the effects of which were significantly reversed by Met treatment. Third, the effects of Met on cardiomyocytes with HG-induced injury, such as the Met-mediated promotion of AKT and GSK3 $\beta$ phosphorylation and the regulation of apoptosis-related protein expression, were abolished after treatment with an AKT inhibitor. Therefore, Met-mediated reductions in diabetesinduced apoptosis might be attributable to activation of the PK2-mediated AKT/GSK3 $\beta$ pathway.

\section{Limitations of the Experiment}

Our current studies have elucidated the important role of PK2 signaling cascade in DCM and Met ameliorates HG-induced cardiac damage through the PK2-mediated AKT/GSK3 $\beta$ pathway using in vivo diabetes animal models and in vitro $\mathrm{HG}$-exposed cardiomyocytes. Any conclusions about the exact role of the Met activated PK2-AKT signaling cascade in DCM must be taken with caution. As proof of concept evidence is still lacking from genetically engineered mouse models of PK2 and AKT, it is quite challenging to better understand the role of PK2 signaling in biological process.

In summary, our data suggest that PK2 may participate in pathophysiological changes in DCM and that Met protects against diabetes-induced cardiac and remodeling, apoptosis, and dysfunction via activation of the $\mathrm{PK} 2 / \mathrm{PKR}$ signaling, thereby regulating the AKT/GSK3 $\beta$ pathway. These findings suggest new strategies for the

\section{REFERENCES}

Aberle, N. S., Lee, K. J., and Ren, J. (2004). Korean anti-inflammatory compound allergina enhances cardiac contractile function in isolated ventricular cardiomyocytes. J. Altern. Complement. Med. 10, 514-518. doi: 10.1089/ 1075553041323858

Agrawal, R. P., Sharma, P., Pal, M., Kochar, A., and Kochar, D. K. (2006). Magnitude of dyslipedemia and its association with micro and macro vascular complications in type 2 diabetes: a hospital based study from Bikaner (Northwest India). Diabetes Res. Clin. Pract. 73, 211-214. doi: 10.1016/j.diabres. 2006.01.006

Arora, H., Boulberdaa, M., Qureshi, R., Bitirim, V., Gasser, A., Messaddeq, N., et al. (2016). Prokineticin receptor-1 signaling promotes epicardial to mesenchymal transition during heart development. Sci. Rep. 6:25541. doi: 10.1038/srep 25541

Bi, Y., Zhang, Y., and Ren, J. (2020). Phosphoinositide 3-Kinase therapy in diabetic cardiomyopathy: unravelling an enigma. Am. J. Physiol. Heart Circ. Physiol. 13:160. doi: 10.1152/ajpheart.00160. 2020

Boulberdaa, M., Turkeri, G., Urayama, K., Dormishian, M., Szatkowski, C., Zimmer, L., et al. (2011a). Genetic inactivation of prokineticin receptor-1 leads to heart and kidney disorders. Arterioscler. Thromb. Vasc. Biol. 31, 842-850. doi: 10.1161/ATVBAHA.110.222323

Boulberdaa, M., Urayama, K., and Nebigil, C. G. (2011b). Prokineticin receptor 1 (PKR1) signalling in cardiovascular and kidney functions. Cardiovasc. Res. 92, 191-198. doi: 10.1093/cvr/cvr228 prevention and treatment of DCM, which are worthy of further clinical study.

\section{DATA AVAILABILITY STATEMENT}

The raw data supporting the conclusions of this article will be made available by the authors, without undue reservation, to any qualified researcher.

\section{ETHICS STATEMENT}

The animal study was reviewed and approved by the Institutional Animal Care and Use Committee at Hubei University of Science and Technology.

\section{AUTHOR CONTRIBUTIONS}

$\mathrm{ZY}, \mathrm{MW}, \mathrm{YZ}, \mathrm{FC}$, and BJ performed the experiments. WZ and WY contributed to analysis and interpretation of the data, and responsible for critically revising the manuscript. All authors read and approved the final manuscript.

\section{FUNDING}

This work was supported by grants from the National Natural Science Foundation of China (81500296 and 81870576), Xianning City of Science and Technology Fund Project (201852), and Research Innovation Team Project of Hubei University of Science and Technology (2018).

Ceylan Isik, A. F., Fliethman, R. M., Wold, L. E., and Ren, J. (2008). Herbal and traditional Chinese medicine for the treatment of cardiovascular complications in diabetes mellitus. Curr. Diabetes Rev. 4, 320-328. doi: 10.2174/ 157339908786241142

Chen, W. S., Xu, P. Z., Gottlob, K., Chen, M. L., Sokol, K., Shiyanova, T., et al. (2001). Growth retardation and increased apoptosis in mice with homozygous disruption of the Aktl gene. Genes Dev. 15, 2203-2208. doi: 10.1101/gad. 913901

Curtis, V. F., Wang, H., Yang, P., McLendon, R. E., Li, X., Zhou, Q. Y., et al. (2013). A PK2/Bv8/PROK2 antagonist suppresses tumorigenic processes by inhibiting angiogenesis in glioma and blocking myeloid cell infiltration in pancreatic cancer. PLoS One 8:e54916. doi: 10.1371/journal.pone.0054916

De Los Santos, S., García-Pérez, V., Hernández-Reséndiz, S., Palma-Flores, C., González-Gutiérrez, C. J., Zazueta, C., et al. (2017). (-)-Epicatechin induces physiological cardiac growth by activation of the PI3K/Akt pathway in mice. Mol. Nutr. Food Res. 61:343. doi: 10.1002/mnfr.201600343

Dormishian, M., Turkeri, G., Urayama, K., Nguyen, T. L., Boulberdaa, M., Messaddeq, N., et al. (2013). Prokineticin receptor-1 is a new regulator of endothelial insulin uptake and capillary formation to control insulin sensitivity and cardiovascular and kidney functions. J. Am. Heart Assoc. 2:e000411. doi: 10.1161/JAHA.113.000411

Foretz, M., Guigas, B., and Viollet, B. (2019). Understanding the glucoregulatory mechanisms of metformin in type 2 diabetes mellitus. Nat. Rev. Endocrinol. 15, 569-589. doi: 10.1038/s41574-019-0242-2

Gao, Y., Yang, Z. G., Ren, Y., Liu, X., Jiang, L., Xie, L. J., et al. (2019). Evaluation of myocardial fibrosis in diabetes with cardiac magnetic resonance T1-mapping: 
correlation with the high-level hemoglobin A1c. Diabetes Res. Clin. Pract. 150, 72-80. doi: 10.1016/j.diabres.2019.03.004

Gardiner, J. V., Bataveljic, A., Patel, N. A., Bewick, G. A., Roy, D., Campbell, D., et al. (2010). Prokineticin 2 is a hypothalamic neuropeptide that potently inhibits food intake. Diabetes Metab. Res. Rev. 59, 397-406. doi: 10.2337/db091198

Gasser, A., Brogi, S., Urayama, K., Nishi, T., Kurose, H., Tafi, A., et al. (2015). Discovery and cardioprotective effects of the first non-Peptide agonists of the G protein-coupled prokineticin receptor-1. PLoS One 10:e0121027. doi: 10.1371/ journal.pone.0121027

Gordon, R., Neal, M. L., Luo, J., Langley, M. R., Harischandra, D. S., Panicker, N., et al. (2016). Prokineticin-2 upregulation during neuronal injury mediates a compensatory protective response against dopaminergic neuronal degeneration. Nat. Commun. 7:12932. doi: 10.1038/ncomms12932

Jafri, A., Siddiqui, S., Rais, J., Ahmad, M. S., Kumar, S., Jafar, T., et al. (2019). Induction of apoptosis by piperine in human cervical adenocarcinoma via ROS mediated mitochondrial pathway and caspase-3 activation. EXCLI J. 18, 154-164. doi: 10.17179/excli2018-1928

Kurebayashi, H., Goi, T., Shimada, M., Tagai, N., Naruse, T., Nakazawa, T., et al. (2015). Prokineticin 2 (PROK2) is an important factor for angiogenesis in colorectal cancer. Oncotarget 6, 26242-26251. doi: 10.18632/oncotarget.4385

Landa-Galvan, H. V., Rios-Castro, E., Romero-Garcia, T., Rueda, A., and Olivares-Reyes, J. A. (2020). Metabolic syndrome diminishes insulin-induced Akt activation and causes aredistribution of Akt-interacting proteins in cardiomyocytes. PLoS One 15:e0228115. doi: 10.1371/journal.pone.0228115

Liu, Y., Yang, Z., Kong, D., Zhang, Y., Yu, W., and Zha, W. (2019). Metformin ameliorates testicular damage in male mice with streptozotocin-induced type 1 diabetes through the PK2/PKR pathway. Oxid. Med. Cell. Longev 18:5681701. doi: 10.1155/2019/5681701

Long, L., Qiu, H., Cai, B., Chen, N., Lu, X., Zheng, S., et al. (2018). Hyperglycemia induced testicular damage in type 2 diabetes mellitus rats exhibiting microcirculation impairments associated with vascular endothelial growth factor decreased via PI3K/Akt pathway. Oncotarget 9, 5321-5336. doi: 10.18632/oncotarget.23915

Maftei, D., Marconi, V., Florenzano, F., Giancotti, L. A., Castelli, M., Moretti, S., et al. (2014). Controlling the activation of the Bv8/prokineticin system reduces neuroinflammation and abolishes thermal and tactile hyperalgesia in neuropathic animals. Br. J. Pharmacol. 171, 4850-4865. doi: 10.1111/bph.12793

Martin, C., Balasubramanian, R., Dwyer, A. A., Au, M. G., Sidis, Y., Kaiser, U. B., et al. (2011). The role of the prokineticin 2 pathway in human reproduction: evidence from the study of human and murine gene mutations. Endocr. Rev. 32, 225-246. doi: 10.1210/er.2010-0007

Mollay, C., Wechselberger, C., Mignogna, G., Negri, L., Melchiorri, P., Barra, D., et al. (1999). Bv8, a small protein from frog skin and its homologue from snake venom induce hyperalgesia in rats. Eur. J. Pharmacol. 374, 189-196. doi: 10.1016/s0014-2999(99)00229-0

Naicker, N., Nagiah, S., Phulukdaree, A., and Chuturgoon, A. (2016). Trigonella foenum-graecum seed extract, 4-hydroxyisoleucine, and metformin stimulate proximal insulin signaling and increase expression of glycogenic enzymes and GLUT2 in HepG2 cells. Metab. Syndr. Relat. Disord. 14, 114-120. doi: 10.1089/ met.2015.0081

Neal, M., Luo, J., Harischandra, D. S., Gordon, R., Sarkar, S., Jin, H., et al. (2018). Prokineticin-2 promotes chemotaxis and alternative A2 reactivity of astrocytes. Glia 66, 2137-2157. doi: 10.1002/glia.23467

Neglia, D., De Caterina, A., Marraccini, P., Natali, A., Ciardetti, M., Vecoli, C., et al. (2007). Impaired myocardial metabolic reserve and substrate selection flexibility during stress in patients with idiopathic dilated cardiomyopathy. Am. J. Physiol. Heart Circ. Physiol. 293, H3270-H3278. doi: 10.1152/ajpheart.00887. 2007

Negri, L., Lattanzi, R., Giannini, E., Canestrelli, M., Nicotra, A., and Melchiorri, P. (2009). Bv8/Prokineticins and their receptors: a new pronociceptive system. Int. Rev. Neurobiol. 85, 145-157. doi: 10.1016/S0074-7742(09)85011-3

Ogurtsova, K., da Rocha Fernandes, J. D., Huang, Y., Linnenkamp, U., Guariguata, L., Cho, N. H., et al. (2017). IDF diabetes atlas: global estimates for the prevalence of diabetes for 2015 and 2040. Diabetes Res. Clin. Pract. 128, 40-50. doi: 10.1016/j.diabres.2017.03.024

Qi, X., Liu, H., Mao, L., Sun, P., and Kong, D. (2017). Combination of exendin-4 and DPP-4 silencing promoted angigenesis of human cornary artery endothelial cells via acrivation of PI3K/Akt pathway. Pak. J. Pharm. Sci. 30, 555-560.
Rubler, S., Dlugash, J., Yuceoglu, Y. Z., Kumral, T., Branwood, A. W., and Grishman, A. (1972). New type of cardiomyopathy associated with diabetic glomerulosclerosis. Am. J. Cardiol. 30, 595-602. doi: 10.1016/0002-9149(72) 90595-4

Sanit, J., Prompunt, E., Adulyaritthikul, P., Nokkaew, N., Mongkolpathumrat, P., Kongpol, K., et al. (2019). Combination of metformin and p38 MAPK inhibitor, SB203580, reduced myocardial ischemia/reperfusion injury in non-obese type 2 diabetic Goto-Kakizaki rats. Exp. Ther. Med. 18, 2701-2714. doi: 10.3892/etm. 2019.7763

Su, G., Sun, G., Liu, H., Shu, L., Zhang, E., and Liang, Z. (2020). Prokineticin a relieves hypoxiaoxia/reoxygenation-induced injury through activation of Akt/mTOR pathway in H9c2 cardiomyocytes. Artif. Cells Nanomed. Biotechnol. 48, 345-352. doi: 10.1080/21691401.2019.1709850

Thandavarayan, R. A., Giridharan, V. V., Watanabe, K., and Konishi, T. (2011). Diabetic cardiomyopathy and oxidative stress: role of antioxidants. Cardiovasc. Hematol. Agents Med. Chem. 9, 225-230. doi: 10.2174/187152511798120877

Urayama, K., Guilini, C., Messaddeq, N., Hu, K., Steenman, M., Kurose, H., et al. (2007). The prokineticin receptor-1 (GPR73) promotes cardiomyocyte survival and angiogenesis. FASEB J. 21, 2980-2993. doi: 10.1096/fj.07-8116com

von Scholten, B. J., Hansen, T. W., Goetze, J. P., Persson, F., and Rossing, P. (2015). Glucagon-like peptide 1 receptor agonist (GLP-1 RA): long-term effect on kidney function in patients with type 2 diabetes. J. Diabetes Complicat. 29, 670-674. doi: 10.1016/j.jdiacomp.2015.04.004

Wang, C. Y., Li, X. D., Hao, Z. H., and Xu, D. (2016). Insulin-like growth factor-1 improves diabetic cardiomyopathy through antioxidative and antiinflammatory processes along with modulation of Akt/GSK-3 $\beta$ signaling in rats. Korean J. Physiol. Pharmacol. 20:613. doi: 10.4196/kjpp.2016.20.6.613

Wang, Y., Guo, X., Ma, H., Lu, L., and Zhang, R. (2016). Prokineticin2 is associated with metabolic syndrome in a middle-aged and elderly Chinese population. Lipids Health Dis. 15:1. doi: 10.1186/s12944-0150172-5

Wang, Y., Feng, W., Xue, W., Tan, Y., Hein, D. W., Li, X. K., et al. (2009). Inactivation of GSK-3beta by metallothionein prevents diabetes-related changes in cardiac energy metabolism, inflammation, nitrosative damage, and remodeling. Diabetes Metab. Res. Rev. 58, 1391-1402. doi: 10.2337/db08-1697

Wold, L. E., Ceylan-Isik, A. F., and Ren, J. (2005). Oxidative stress and stress signaling: menace of diabetic cardiomyopathy. Acta Pharmacol. Sin. 26, 908917. doi: 10.1111/j.1745-7254.2005.00146.x

Xin, H., Lu, R., Lee, H., Zhang, W., Zhang, C., Deng, J., et al. (2013). G-proteincoupled receptor agonist BV8/prokineticin-2 and STAT3 protein form a feedforward loop in both normal and malignant myeloid cells. J. Biol. Chem. 288, 13842-13849. doi: 10.1074/jbc.M113.450049

Xu, H., Zhou, Y., Liu, Y., Ping, J., Shou, Q., Chen, F., et al. (2016). Metformin improves hepatic IRS2/PI3K/Akt signaling in insulin-resistant rats of NASH and cirrhosis. J. Endocrinol. 229, 133-144. doi: 10.1530/JOE-150409

Yang, F., Qin, Y., Wang, Y., Meng, S., Xian, H., Che, H., et al. (2019). Metformin inhibits the NLRP3 inflammasome via AMPK/mTOR-dependent effects in diabetic cardiomyopathy. Int. J. Biol. Sci. 15, 1010-1019. doi: 10.7150/ijbs.29680

Yu, W., Zha, W., Ke, Z., Min, Q., Li, C., Sun, H., et al. (2016). Curcumin protects neonatal rat cardiomyocytes against high glucose-induced apoptosis via PI3K/Akt signalling pathway. J. Diabetes Res. 2016:4158591. doi: 10.1155/ 2016/4158591

Zhang, Y., Babcock, S. A., Hu, N., Maris, J. R., Wang, H., and Ren, J. (2012). Mitochondrial aldehyde dehydrogenase (ALDH2) protects against streptozotocin-induced diabetic cardiomyopathy: role of GSK3 $\beta$ and mitochondrial function. BMC Med. 10:40. doi: 10.1186/1741-7015$10-40$

Conflict of Interest: The authors declare that the research was conducted in the absence of any commercial or financial relationships that could be construed as a potential conflict of interest.

Copyright (c) 2020 Yang, Wang, Zhang, Cai, Jiang, Zha and Yu. This is an open-access article distributed under the terms of the Creative Commons Attribution License (CC BY). The use, distribution or reproduction in other forums is permitted, provided the original author(s) and the copyright owner(s) are credited and that the original publication in this journal is cited, in accordance with accepted academic practice. No use, distribution or reproduction is permitted which does not comply with these terms. 